\title{
A Synchrotron Design for Macromolecular Structure Determination
}

\author{
Joseph P. Lidestri*, Columbia University Department of Biochemistry and Molecular Biophysics, \\ 630 West 168th Street New York, NY 10032 USA
}

Abstract

A $2.5 \mathrm{GeV} 200 \mathrm{~mA}$ synchrotron has been conceptually designed with superconducting bend magnets and superconducting RF cavities. The preliminary design optimizes the synchrotron parameters for protein structure determination by considering both the acceptance phase space and the radiation flux limit of a typical protein crystal. By exploiting 5 Tesla superconducting dipoles and 500Mhz superconducting RF cavities the synchrotron can be facilitated within a 20 meter diameter. The periodic magnet lattice utilizes 8 double bend achromat (DBA) cells to produce an $\mathrm{x}$-ray source with a brightness typical of second generation storage rings. The synchrotron radiation from the 5 Tesla bends would have a spectral brightness of $2.1 \times 10^{14}$ photons $/ \mathrm{s} / \mathrm{mr}^{2} / \mathrm{mm}^{2} /(0.1 \%$ bandwidth $)$ at the K-edge of Selenium which is well suited for multiwavelength anomalous diffraction (MAD) measurements [1].

\section{INTRODUCTION}

Synchrotron radiation was utilized at the DESY synchrotron in Hamburg as early as 1970 to measure x-ray diffraction from a slice of insect flight muscle. Biological crystallographers quickly recognized the need to develop analytical methods to explore the atomic detail of biological macromolecules with synchrotron radiation. At that time advances in phasing methods and instrumentation lagged behind while synchrotrons were being developed into the supreme light source for $\mathrm{x}$-ray research. One of the main advances in $\mathrm{x}$-ray analysis techniques responsible for the wide spread use of synchrotrons in structural biology as been the development of the phasing method called multiwavelength anomalous diffraction (MAD). MAD is a technique that can directly solve the phasing problem from a single crystal sample but requires the collection of diffraction data at multiple $\mathrm{x}$-ray energies. The typical MAD experiment is conducted with selenomethionyl proteins at $\mathrm{x}$-ray energies above, below and including the K-edge of selenium. Due to the recent advances that have taken place such as the development of MAD, macromolecular crystallography has matured into a powerful technique that is expected sustain structural biology for many years to come. The steady growth of synchrotron research has lead to the construction of approximately 50 synchrotrons worldwide with macromolecular crystallography being the fastest growing analytic technique pursued. At present 27 synchrotron have or plan to facilitate at least one $\mathrm{x}$-ray beam line to exploit methods in protein structure determination. While in the past compact accelerator have been

*HHMI, lidestri@convex.hhmi.columbia.edu conceptually designed using insertion devices as radiation sources this paper reviews a ring design based on superconducting bend magnets [2] that would produce synchrotron radiation from 16 arc sources dedicated to the crystallographic determination of biological macromolecule structures.

\section{DESIGN CRITERIA}

The accelerator parameters for this design are optimized to produce the required synchrotron radiation parameters typically needed for macromolecular crystallography. The three criteria used include 1)the acceptance phase space of a crystal 2)the radiation damge limit of a crystal 3)the energy and energy resolusion requirements of the synchrotron source.

(Phase Space Analysis) Phase space analysis has long been used by accelerator physicists to study charge particle beam dynamics. Due to the unique properties of synchrotron radiation phase space analysis was then applied to $\mathrm{x}$-ray optics to match a synchrotron source to optics. The most common synchrotron metric used for flux is in terms of phase space volume. By definition [6] flux per unit of phase space volume is called Brightness expressed in the conventional units photons $/ \mathrm{s} / \mathrm{mr}^{2} / \mathrm{mm}^{2} /(0.1 \%$ bandwidth). This is a good unit to keep track of in an optical design since the Brightness of an image cannot exceed that of the source and can only be equal to it if losses in the optical system are negligible. Since the volume of source phase space is invariant under optical manipulation this unit can be misleading. To best define the source parameters it is useful to decompose the source phase space volume into separate vertical and horizontal phase planes. By decomposing Brightness into two phase planes a projected source can be optimally matched to a desired image. A crystal sample can also be described by two phase planes that characterize the crystals angular and spacial acceptance of radiant flux. The crystal AcceptancePhaseSpace can be used to define an image of the projected synchrorton source as in Fig.1. As seen in Fig.1 the angular acceptance for a crystal is determined by its mosaicity and can range from $(2-18) \mathrm{mrad}$. When protein crystals are frozen from $290 \mathrm{~K}$ to $100 \mathrm{~K}$ for radiation protection rocking witdth typically increase by a factor of ten. The spatial axis of the acceptance pahse plane of a crystal is simply the size of the crystal which can range from 25 to $300 \mathrm{um}$. As illustrated in Fig. 1 the rotation of the horizontal axis which is the typical method results in spreading out the phase plane in the vertical plane. For this reason it is a good idea to use (2-5) mrad as the range of angular acceptance so that low divergence data can be collected on crystals with high $18 \mathrm{mrad}$ 
mosaicity. The ideal image phase planes can be defined by back projecting the crystal accepatnce phase planes and the source phase planes can be defined by back projecting the image planes. It will be assumed in designing the source that the eccentricity of the phase plane ellipse associated with the gaussian source can be manipulated be a factor of 4 with conventional optical schemes.
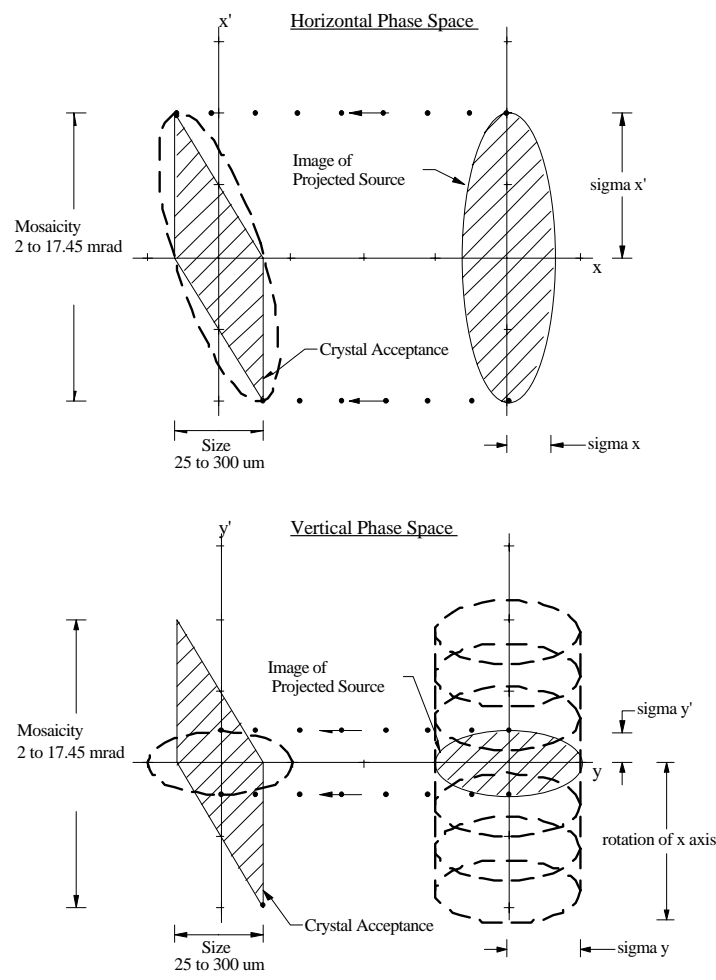

Figure 1: Acceptance phase space of typical protein crystal with matching source image.

(Radiation Damage Limit) Radiation damage to protein sample has recently been studied [3] by characterizing non-isomorphism introduced by $\mathrm{x}$-rays. Radiation damage induced changes have been observed at flux densities $\geq 7 \times 10^{13}$ photons $/ \mathrm{s} / \mathrm{mm}^{2}$. This flux density was from a (13.1-13.3keV) incident beam and showed to be in close agreement with the absorbed dose limits previously established by R.Henderson. The integrated dose model of radiation damage fails to explain the equilibrium state were cryogenic samples $(\approx 100 K)$ seem to survive indefinitely at flux densities $\leq 1 \times 10^{13}$ photons $/ \mathrm{s} / \mathrm{mm}^{2}$. An equilibrium state seems to suggest that energy is tranferred out of the crystal sample in the form of heat and charge. Such an equilibrium state implies a flux rate dependency that would require the consideration of the peak flux density time history of the x-ray source. The criteria used for this synchrotron design will be the average flux density $\leq 1 \times 10^{13}$ photons $/ \mathrm{s} / \mathrm{mm}^{2}$ for survival.

(Energy Resolusion Requirement) The energy requirement chosen for this analysis is based on the needs of a typical MAD experiment with a selenomethionyl protein. An energy centered at $12.658 \mathrm{keV}$ with better that $10 \mathrm{eV}$ energy resolusion as well as tunability is required. Energy selection is accomplished with a monochromator by matching the xray beam divergence to the desired energy resolusion. For a nondispersive monochromator and a polychromatic $\mathrm{x}$-ray source the energy resolusion can be obtained from eq. 1

$$
d E=E \cot \theta d \theta
$$

Therefore to keep the dispersion from a Silicon(111) monomchromator below $10 \mathrm{eV}$ only $125 \mathrm{urad}$ angular divergence of the incoming beam can be accepted

\section{ACCELERATOR PARAMETERS}

- Ring Circumference $=63.14 \mathrm{~m}$

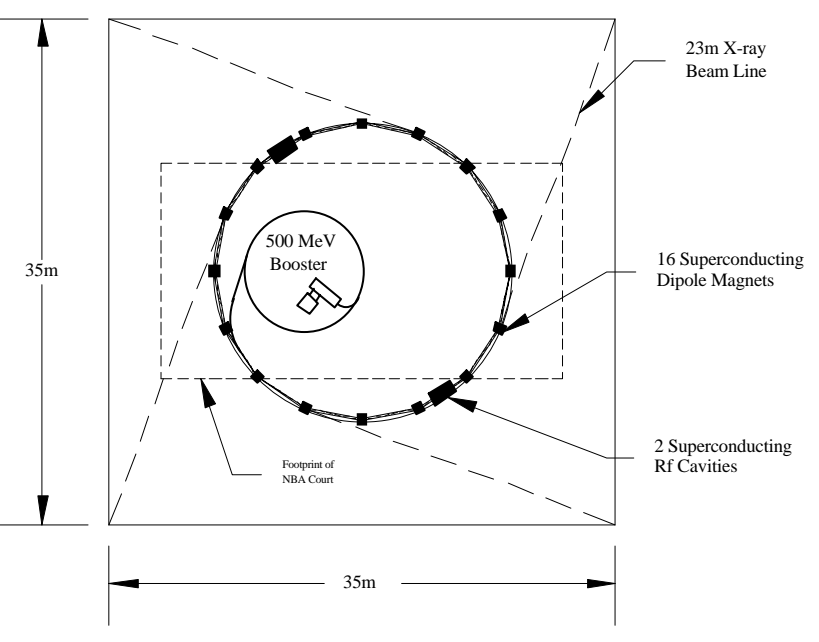

Figure 2: Layout of Synchrotron Site $2.500 \mathrm{GeV}, 200 \mathrm{~mA}$

(Injection) The injection energy for the storage ring is $500 \mathrm{MeV}$ and is base on the reliable booster synchrotron used at the ANKA [4] facility in Karlsruhe. A $70 \mathrm{keV}, 500 \mathrm{~mA}$ electron gun is used as a source for a 10 turn $3 \mathrm{GHz}$ microtron that injects $10 \mathrm{~mA}$ of $53 \mathrm{MeV}, 10 \mathrm{us}$ pulses of electrons into a booster synchrotron. The $26.4 \mathrm{~m}$ circumference booster accelerates the $53 \mathrm{MeV}$ electrons up to the $500 \mathrm{MeV}$ at which point it ready to inject at a rate of $1 \mathrm{~Hz}$. Many of the systems that have developed at ANKA are planned to be utilized in this design such as kicker and septum magents as well as other focussing magnets.

(Superconducting $\mathrm{Rf}$ ) The $413.710 \mathrm{~kW}$ of total radiation power along with an energy loss/turn $=2.069 \mathrm{MeV}$ requires the use of 2 superconducting Rf cavities. The cavities will be the $500 \mathrm{MHz}$ Cornell design [5] that are now commercially available. $600 \mathrm{~kW}$ of Klystron power will be 
needed to power the 2 cavities and a desired overvoltage factor of 2 requires a total Rf cavity voltage of 4137.00 .

(Superconducting Bends) The bend magnets for this ring design are based on the Lawrence Berkely National Laboratory Superbend technology [4]which incorporates conduction-cooled $\mathrm{Nb}$-Ti windings. The dipole magnet should have a bend radius of $\rho=1.668 \mathrm{~m}$ and field strength $=5 T$ esla corresponding to a Rigidity $=8.339 \mathrm{Tm}$.

(Double-Bend-Achromat) The ring geometry is made up of 4 superperiods each of which contain 2double bend achromat cells. In order to optimize this lattice a simple DBA cell was analyzed with 2 bends and a single focusing quadrupole midway between the dipoles. The following achromaticity condition [6] was used for this simple cell to determine the quadrupole focussing strength.

$$
\rho \tan \frac{\theta}{2+l}=\frac{1}{\sqrt{k}} \cot \frac{\sqrt{k} l_{q}}{2}
$$

A quadrupole strength of $4.9\left(1 / \mathrm{m}^{2}\right)$ was determined for the following cell parameters, bend radius $=1.668 \mathrm{~m}$, bend deflecting angle $=22.5 \mathrm{deg}$, quadrupole length $=$ $0.320 \mathrm{~m}$, drift space $=0.890 \mathrm{~m}$.

(Periodic Lattice Structure) Two qaudrupole doublets were then put symmetrically outside the dispersion matching section and two sextupole doublets were but inside the dispersion matching section for chromatic correction to complete the cell. This DBA cell was simulated with the matrix code Particle Beam 2000 [7]. The simulations were used to find the periodic solution with the minimum beam emittance. Once the solution was found one of the low dispersion drift sections were removed and then resymmetrized to complete one superperiod. Four superperiods complete the ring and the periodic solution found had a horizontal emittance, $\epsilon_{x}=38.516 \mathrm{~nm}-\mathrm{rad}$ and vertical emittance, $\epsilon_{y}=385.155 \mathrm{pm}$. The ideal minimum emittance can be calculated with the following analytic expression

$$
\begin{gathered}
\epsilon_{d b a}=\frac{C_{q} \gamma^{2} \theta^{3}}{4 \sqrt{15}} \\
\epsilon_{d b a}(r a d m)=5.036 \times 10^{-13} E^{2}\left(\mathrm{GeV}^{2}\right) \theta^{3}\left(\mathrm{deg}^{3}\right)
\end{gathered}
$$

The calculated minimum is $\epsilon_{x}=35.85 \mathrm{~nm}-\mathrm{rad}$ which is in close agrees well with the above results.

(Beam Dynamic Functions at The Middle Of Bend) Betatron Function $\beta_{x}=0.204 m, \beta_{y}=12.416 \mathrm{~m}$ Dispersion Functions $\eta_{x}=0.037 m, \eta_{x^{\prime}}=-0.042 \alpha_{x}=-\beta_{x}^{\prime} / 2=$ $0.075, \alpha_{y}=-\beta_{y}^{\prime} / 2=2.599 \gamma_{x}=\left(1+\alpha_{x}\right) / \beta_{x}, \gamma_{y}=$ $\left(1+\alpha_{y}\right) / \beta_{y}$

\section{SYNCHROTRON RADIATION}

The 16 arc sources will have critical photon energy, $e_{\text {crit }}=20.789 \mathrm{KeV}$ corresponding to a wavelength, $\lambda_{\text {crit }}=0.564 \AA$.

(Source Phase Space) The source Size, $\sigma_{x}=$ 108.69um, $\sigma_{y}=69.14 u m$ Source Divergence, $\sigma_{x^{\prime}}=$

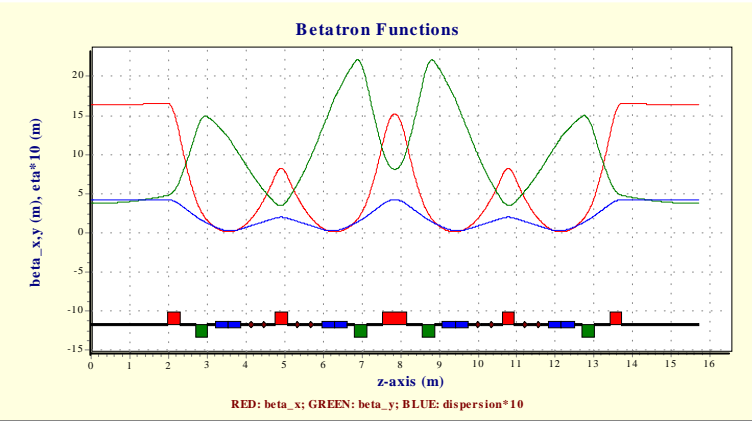

Figure 3:

441.55urad, $\sigma_{y^{\prime}}=15.51$ urad Vertical Opening Angle, $\sigma_{\psi}=0.172 \mathrm{mrad}$ horizontal opening angle $\sigma_{\theta}=$ $0.5988 \mathrm{mrad} / \mathrm{mm}$ of arc

(Source Brightness) The total radiated flux is $6.396 \times$ $10^{16}$ photons $/ \mathrm{s}$ integrated over the total source area $718 \mathrm{~mm}^{2}$. The Figure 4 is a plot of the Spectral Brightness as a function of photon energy. Spectral Bright-

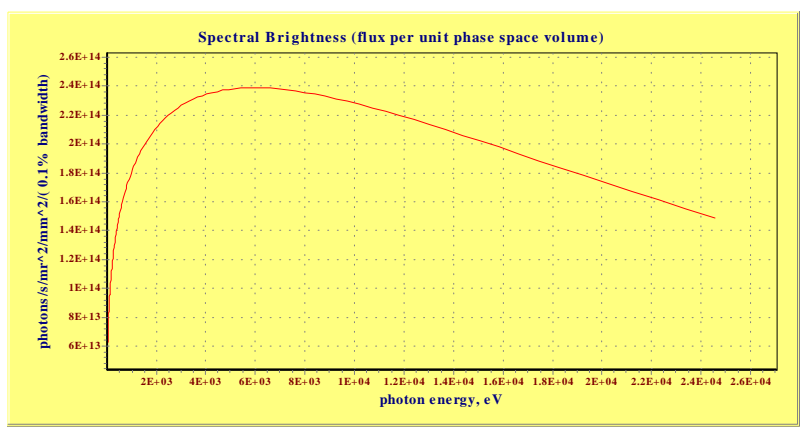

Figure 4: Spectral brightness of $1 \mathrm{mr}$ of arc in horizontal plane.

ness at the K-edge of Selenium $(12.658 \mathrm{KeV})$ is $2.1 \times$ $10^{14}$ photons $/ \mathrm{s} / \mathrm{mr}^{2} / \mathrm{mm}^{2} /(0.1 \%$ bandwidth $)$

\section{REFERENCES}

[1] Hendrickson WA.,[Review] [48 refs] Science. 254(5028):518, 1991 Oct 4.

[2] Zbanik,J.;Wang,S.T.;et al.A IEEE Transactions on Applied Superconductivity 2000,11 2531,2001.

[3] Ravelli RB. McSweeney SM. Structure. 8(3):315-28, 2000 15.

[4] Einfeld, D.; Pont, M., The injector of the storage ring ANKA, Nachr.2000.

[5] H. Padamsee, et al.,Particle Accelerators,Vol.40,pp.1741(1992).

[6] Wiedemann, H., Particle Accelerator Physics Vol.1, Springer 1993.

[7] Particle Beam 2000 is a matrix code by Helmut Wiedemann at Stanford University that calculates the periodic solution for a magnetic lattice in terms of a $6 \times 6$ tranformation matrix. http://coherent.stanford.edu/He-Web/PC-programs.html 